\title{
АНАЛИЗ ТЕКСТА В УСЛОВИЯХ АВТОМАТИЗАЦИИ ПЕРЕВОДЧЕСКОЙ ДЕЯТЕЛЬНОСТИ
}

\author{
Л. В. Елизарова, О. Ю. Кустова \\ Российский государственный педагогический университет имени А. И. Герцена
}

\section{THE TEXT ANALYSIS IN TRANSLATION PROCESS AUTOMATION}

\author{
L. V. Elizarova, O. Yu. Kustova \\ Herzen State Pedagogical University of Russia
}

\begin{abstract}
Аннотация: статья посвящена исследованию модификации параметров анализа текста в изменивщихся условиях осуществления переводческой деятельности, в частности, с учетом человеко-машинного взаимодействия. Цель исследования, направленная на выявление специфики переводческого анализа, обусловленной потребностями отрасли лингвистических услуг, и формирование современного подхода $к$ развитию аналитической компетенции будущих переводчиков, решается в ходе диахронического и синхронического анализа трактовок понятия «переводческий анализ» в теории и дидактике перевода, описания технологического прочесса письменного перевода в профессиональной сфере и выявления индикаторов аналитической компетенции, востребованных при использовании машинного перевода. Результать исследования имеют теоретическую и практическую значимость, так как, с одной стороны, в статье уточняется понятие переводческого анализа текста, актуальное для теории перевода, по-новому определяются функиии и содержание аналитических действий переводчика; с другой стороны, выработка нового подхода к обучению будущих переводчиков в части формирования и развития аналитической компетенции важна как для подготовки специалистов, востребованных в переводческой отрасли, так и для преодоления определенных противоречий, имеющихся между научным и профессиональным сообществами относительно необходимости обучения переводческому анализу текста. Современное понимание переводческого анализа текста обусловлено взаимодействием разнородных факторов прочесса перевода, каждый из которых по-своему влияет на когнитивную деятельность переводчика как языковойличности: использование технологий машинного перевода, постредактирование машинного перевода, создание контролируемого языка, взаимодействие специллистов разного профиля в производственном процессе (менеджер проекта, переводчик, редактор, специалист по контролю качества перевода), изменение модели прочесса перевода в связи с использованием компьютерных технологий, актуализация аналитических действий переводчика на всех этапах осуществления письменного перевода, а не только на этапе подготовки к переводу.
\end{abstract}

Ключевые слова: переводческий анализ текста, машинный перевод, компетенции переводчика, языковая личность переводчика, контролируемый язык.

Abstract: this article deals with the research of the modification of text analysis parameters in the changed conditions of the translation process, taking into account the human-machine interaction. The aim of this paper is to identify specific conditions of the translation analysis which are based on the needs of language services, to create a new approach to development of the analytical competence of future translators. The study is carried out in the course of the diachronic and synchronic analysis of the concept "translation analysis" in the theory and didactics of translation, description of the technological process of translation in the professional field and identification of indicators of analytical competence relevant for machine translation. The findings of the study are of theoretical and practical importance, since, on the one hand, the article clarifies the concept of translation analysis of the text relevant for the theory of translation, defines the translator's analytical actions and their functions in a new way; on the other hand, the development of a new approach to future translators training in terms of the formation and development of analytical competence is important both for specialists training who are in demand in the translation industry and for overcoming certain contradictions between the scientific and professional

(C) Елизарова Л. В., Кустова О. Ю., 2021

Контент доступен под лицензией Creative Commons Attribution 4.0 License.

The content is available under Creative Commons Attribution 4.0 License. 
communities regarding the need for training in the translation text analysis. The contemporary understanding of the text analysis of the translation is due to the combination of various factors of the translation process, each of which in its own way affects the cognitive activity of the translator as a linguistic personality: the use of machine translation technologies, post-editing of the machine translation, creation of a controlled language, interaction of various specialists in the translation process (a project manager, translator, editor, translation quality control specialist), changing of the model of the translation process regarding the use of computer technologies, updating the translator's analytical actions at all stages of the translation process, not only at the stage of preparation for translation.

Key words: translation text analysis, machine translation, translator's competence, translator's linguistic personality, controlled language.

\section{Введение}

Несмотря на то, что значимость переводческого анализа как одной из процедур процесса перевода обоснована в научной литературе, а его способность повышать качество переводного текста не подвергается сомнению, среди представителей переводческой отрасли преобладает скептическое отношение к данной форме деятельности. Анализ текста считается прерогативой и «излишеством» академического образования в области лингвистики, мало корреспондирующим с переводческой практикой в условиях реальной деятельности, а затраты времени на него в условиях машинного перевода видятся нецелесообразными. Таким образом, переводческий анализ становится одним из тех противоречий, которые наблюдаются в представлениях научного сообщества и работодателей относительно подготовки молодых специалистов.

Однако если внимательно рассмотреть особенности организации процесса перевода в современной компании и проанализировать специфику деятельности отдельных специалистов, отвечающих за подготовку качественного продукта перевода, можно сделать вывод, что переводческий анализ текста является востребованной и необходимой частью профессиональной деятельности в сфере оказания лингвистических услуг. В частности, в этом можно убедиться на основании сравнительного анализа оценки необходимых переводчику компетенций со стороны представителей вузов и переводческой отрасли, который был проведен Александром Бондаренко. Основной вывод данного исследования подтверждает общую тенденцию: «зачастую результаты оценки компетенций одной стороной кардинально отличаются от оценок другой: большинство высоко оцененных компетенций одной группы опрошенных встретили неодобрение (или, по крайней мере, не такую высокую оценку) другой» [1, с. 38]. Однако, несмотря на то, что умение анализировать тексты не оценивалось в исследовании А. Бондаренко как отдельное требование, стоит обратить внимание на те компетенции выпускников, которые были выделены как наиболее значимые именно представителями переводческой отрасли. Среди них «способность переводить специ- ализированные тексты, относящиеся к одной и более сферам; способность переводить тексты низкого качества, с грамматическими, стилистическими и другими ошибками» [1, с. 192]. Данные способности предполагают наличие сформированной аналитической компетенции у переводчика, т. е. даже если анализ текста не осознается как отдельная компетенция, он является необходимой составляющей действий практикующего переводчика. Отсюда можно сделать заключение об имплицитном характере компетенции, которая включается во все формы человеко-машинного взаимодействия в условиях современной организации труда переводчика.

Таким образом, в настоящее время необходимо переосмыслить функции и содержательное наполнение переводческого анализа текста и определить, каким образом и в каком объеме востребованы человеческие мыслительные операции, связанные с анализом текста, при использовании машинного перевода. Целью данной статьи является исследование факторов, влияющих на формирование новых практико-ориентированных подходов к переводческому анализу текста.

Для достижения поставленной цели в статье решаются следующие задачи:

1) уточнить понятие переводческого анализа в теории и дидактике перевода;

2) переосмыслить подход к переводческому анализу в условиях автоматизации переводческой деятельности;

3) определить условия использования аналитической компетенции переводчика (и шире - специалиста отрасли лингвистических услуг) и сформировать параметры переводческого анализа текста с опорой на потребности отрасли.

\section{Материалы и методы исследования}

Вопросы переводческого анализа традиционно рассматриваются в разделах переводоведения, направленных на решение задач переводческой практики. В дидактическом аспекте данный анализ понимается как неотьемлемая часть этапа подготовки к переводу, как необходимое условие, «залог» успешного и качественного перевода. При этом ранее в 
соответствующих публикациях по теме наблюдалась тенденция к отождествлению с лингвостилистическим или - в другой терминологии - филологическим анализом текста, а основанием для такого подхода являлась связь с существующими методами анализа. В дальнейшем по мере трансформации переводческих концепций произошло разграничение данных понятий, в то же время их взаимодействие не вызывает сомнения. Исследователи подчеркивают, что задачи литературоведческого или филологического анализа текста и переводческого анализа текста различаются. «Если у первого задача - раскрыть специфику текста и выявить закономерности его построения и языкового оформления для научного исследования и интерпретации, то у второго задача состоит в предварительной подготовительной работе для создания адекватного перевода» [2, с. 2]. Н. А. Каширина отмечает различие между филологическим анализом текста и переводческим анализом, поскольку именно последний позволяет переводчику осуществить выбор необходимого переводческого решения [3, с. 258]. Выбор переводческого решения зависит от многих факторов, совокупность которых и определяет стратегию перевода. Именно в определении стратегии, на наш взгляд, заключается основная задача переводческого анализа. При этом вопрос о выборе какой-либо стратегии представляется достаточно проблематичным: с учетом особенностей современных текстов не может быть выбрана какая-либо одна стратегия. На практике нередко встречаются случаи подмены понятия стратегии каким-либо приемом или способом перевода. Переводческая стратегия как совокупность действий переводчика носит комплексный и одновременно индивидуальный характер, поэтому целесообразно говорить не о выборе, а о выработке стратегии. Изучение проблематики, связанной с выяснением сущности стратегий перевода, способов их формирования и типологии является значимым не только с переводоведческой точки зрения, но и с точки зрения дидактики перевода и заслуживает более детального рассмотрения в других исследованиях.

В целом, на современном этапе, прежде всего в дидактике перевода, цель и задачи переводческого анализа достаточно четко определены, как и его место в процессе перевода - на подготовительном этапе, что нашло отражение в соответствующей терминологии: предпереводческий анализ, предпереводной анализ, text analysis for translation и т. д. Понимая условность деления на этапы перевода, детерминированную характером переводческой деятельности как сложного когнитивного феномена, исследователи в рамках соответствующих подходов связывают проведение переводческого анализа с этапом подготовки к выполнению перевода. Вместе с тем, несмотря на различия предлагаемых концепций, В. Б. Кашкин отмечает определенную общность подходов, так как они базируются на объективных характеристиках внутритекстовой организации и внетекстовых связей. Как для российских, так и зарубежных исследователей отправной точкой переводческого анализа является типология текстов, а основной его целью - глубокое проникновение в содержание исходного текста, обеспечивающее наиболее оптимальную возможность интерпретации средствами переводящего языка [4, с. 50-51]. Дискуссионными остаются вопросы, связанные с уровнями и параметрами переводческого анализа, а также некоторые другие проблемы.

Основным упущением современных трактовок переводческого анализа является отсутствие упоминания о том, что анализ экстра- и интралингвистических факторов с целью выработки переводческой стратегии должен выполняться с учетом технических возможностей осуществления перевода. Если и далее оставлять данный фактор без внимания, разрыв между подготовкой и профессиональной деятельностью переводчиков станет еще более ощутимым. При определении переводческого анализа и его алгоритмов нельзя не учитывать изменившийся характер переводческой деятельности, обусловленный требованиями современного рынка труда, внедрением и широким использованием информационных технологий, прежде всего машинного перевода, а также систем переводческой памяти и т. д. К другим факторам, которые необходимо учитывать при трактовке переводческого анализа, следует отнести изменение модели процесса перевода в связи с эволюцией компьютерных технологий. Если ранее этот процесс был представлен в последовательности «автор - переводчик - получатель», то сейчас в роли переводчика может выступать как человек, так и компьютерная программа, или они могут выполнять функции переводчика совместно. Соответственно сейчас процесс перевода выглядит следующим образом: «автор - переводчик (человек) / переводчик (компьютерная программа) - получатель». Использование средств автоматизации в целях повышения производительности труда, оптимизации временных и финансовых затрат привело к качественным изменениям в работе переводчика: он не генерирует собственные переводческие решения, а осуществляет выбор одного из предлагаемых вариантов перевода [5, с. 493].

Указанные факторы предопределяют необходимость переосмысления переводческого анализа в части его определения, а также пересмотра и дополнения его «технологических» параметров. В современных условиях представляется целесообразным более широкое толкование данного явления, предполагающего активную деятельность переводчика и других специалистов отрасли переводческих услуг, 
отвечающих за качество конечного продукта. Эта деятельность направлена как на глубокое понимание переводимого текста, на определение стратегии его перевода, так и на оценку результатов перевода - на осознание и выявление связи между начальным и конечным этапами перевода. Понимание анализа в условиях автоматизации переводческой деятельности не должно ограничиваться выполнением определенных алгоритмов и действий на этапе подготовки. Анализ текста выполняется и на постпереводческой стадии. В подтверждение такой позиции приведем определение анализа текста, который в явном или интуитивном виде присутствует «как на этапах понимания и преобразования исходного текста, так и на стадии проверки, редактирования и оценки качества результатов перевода» [6, с. 170]. Обзор исследовательских подходов к переводу, в том числе определения перевода как аналитико-синтетического процесca, также позволяет прийти к выводу о тесной связи действий переводчика на подготовительном и заключительном этапах работы над текстом, в связи с чем в процессе обучения будущих переводчиков необходимо формировать и развивать умение видеть связь начального и конечного этапов перевода.

Развитие технологий машинного перевода, а также интегрированных с ним систем переводческой памяти, требует особой схемы переводческого анализа, которая, по мнению исследователей, должна носить машинно-ориентированный характер (термин Никитиной). При этом речь идет не об отказе от лингвостилистической составляющей анализа, а о переосмыслении некоторых из существующих параметров в изменившихся условиях переводческой деятельности. Переводческий анализ предполагает не только знание типологических и других характеристик текста, но и знание особенностей (модели, архитектуры) и возможностей (функций) используемого программного продукта.

В связи с этим возникает вопрос о том, каким же должен быть / как должен выглядеть алгоритм маиинно-ориентированного переводческого анализа. Для ответа на данный вопрос представляется целесообразным обратиться к концепции В. В. Сдобникова об определении понятия «стратегия перевода» на основе рассмотрения таких составляющих процесса формирования стратегии, как ориентирование в ситуации, определение цели деятельности, прогнозирование и планирование [7, с. 165].

Механизм выработки стратегии осуществления деятельности включает уяснение специфики ситуации. В условиях компьютерно-опосредованной коммуникации к наиболее значимым параметрам коммуникативной ситуации можно отнести личность и роль инициатора перевода, цель осуществления перевода, условия осуществления коммуникантами предметной деятельности. Таким образом, «уяснение ситуации», предполагающее определение внешних параметров текста, характеристику автора оригинала и реципиента текста перевода параллельно с выявлением текстотипологических характеристик позволяет установить степень допустимости машинного перевода (или других программ) для текста. Кроме этого, ориентирование в ситуации может быть направлено на выбор переводчиком программы с соответствующими функциональными возможностями для достижения оптимального результата как на пред-, так и на постпереводческом этапах.

Определение цели деятельности представляется значимым параметром, но подвергается в определенной мере переосмыслению. Если в концепции В. В. Сдобникова этот параметр «привносится» в коммуникативную ситуацию самим переводчиком, точнее, становится осознаваемым им только в результате проведения соответствующего анализа [7, с. 168], то в условиях автоматизации перевода цели деятельности в меньшей степени зависят от переводчика. Скорее они детерминированы требованиями заказчика относительно конечного продукта перевода.

В современных условиях выполнения заказа на перевод прогнозирование возможных изменений коммуникативной ситуации и ее дальнейшего развития приобретает особое значение. С опорой на результаты проведенного анализа текста, включающего определение лексических и синтаксических особенностей, стилистических приемов и экспрессивных средств, средств выдвижения информации, принимается решение о (не)возможности адаптации исходного текста для облегчения процесса машинного перевода. При этом очень важно сочетать машинно-ориентированный предпереводческий анализ и редактирование. Эти два этапа обеспечивают эффективный переводческий процесс, когда переводчик прогнозирует, повторно формулирует содержание текста и исправляет перевод [2]. Таким образом, можно говорить о предварительном редактировании оригинального текста, которое может улучшить качество машинного перевода.

В современной литературе с предредактированием связано понятие контролируемого языка, использование технологий которого направлено на упрощение текстов, главным образом, на лексическом и грамматическом уровнях. Следует отметить, что создание контролируемого языка нецелесообразно для отдельного текста/проекта из-за значительных временных затрат и довольно трудоемкого процесса. Оптимально создавать контролируемый язык только в том случае, если он еще не разработан для определенной области, в которой осуществляется перевод, а также, если он будет использоваться не одним специалистом, а группой (например, в компании). 
Только в этом случае будут оправданы затраты и гарантировано пополнение словарной базы, так называемого «контролируемого словаря». Технологии упрощения текста могут полностью исключить этап постредактирования, а их изучение как одной из составляющих переводческого анализа будет ограничено исключительно подготовительным этапом. Тогда алгоритмы переводческого анализа должны быть дополнены параметром контролируемого языка, предусматривающим знание методик разработки правил, ограничивающих использование лексико-синтаксического инвентаря. Данный параметр также должен включать и понятие «индикаторы переводимости» [8], к которым относятся многозначные единицы, сложные синтаксические конструкции, стилистические приемы, экспрессивные средства и т. д.

Прогнозирование доли машинного перевода на первоначальном этапе анализа позволяет наиболее эффективно распределить время, необходимое для перевода. Решение о том, какой объем текста будет представлен для машинного перевода, как эффективнее использовать машинную память и следует ли создавать адаптированный/упрощенный вариант текста для компьютерной программы, может значительно ускорить процесс перевода и минимизировать усилия по окончательному редактированию.

Как мы видим, прогнозирование тесно связано с другим компонентом стратегии перевода - планированием, охватывающим все этапы переводческого процесса. Кроме принятия решения о включении в анализ вышеуказанных действий, планирование предполагает оценку соответствующего программного продукта, его характеристик, а также наличие опыта работы с такой программой для выполнения поставленных задач. С планированием, на наш взгляд, связан еще один параметр переводческого анализа - доминанты или приоритеты перевода. Например, в контексте определенных видов научного общения лингвистическая точность может не быть приоритетом по сравнению, например, с терминологической точностью. Проверка правописания, грамматики и пунктуации может быть не менее важна, нежели техническая точность. В свою очередь, исправление или переформулировка определенных словосочетаний могут повысить лингвистическое качество, но привести к искажению информации. В ходе переводческого анализа переводчик принимает решение об определенных приоритетах, особенно если перевод выполняется в сжатые сроки.

Чтобы точнее определить параметры текстового анализа в условиях автоматизации переводческой деятельности и определить факторы формирования и развития аналитической компетенции (будущего) переводчика в различных формах человеко-машин- ного взаимодействия, необходимо проанализировать потребности отрасли лингвистических услуг и принятые практики организации производства.

Как указывалось выше, состав лингвистических услуг, предлагаемых переводческими компаниями (приводятся обобщенные данные сайтов переводческих компаний Янус, Логрус Глобал, СДЛРус и других), а также описание подходов к применению машинного перевода свидетельствуют о том, что аналитическая компетенция специалистов в полной мере используется в производственном процессе. С помощью переводческого анализа выявляются и/или решаются разнообразные задачи, в частности:

- диагностика категории переводимых материалов, в ходе которой оценивается применимость постредактирования;

- логико-смысловой анализ текста с целью выявления двусмысленности/неоднозначности исходного текста;

- на этапе предредактирования производится стандартизация и упрощение конструкций на основе знаний об особенностях движка машинного перевода, проверка грамматической, орфографической и логической правильности исходного текста;

- на этапе постредактирования происходит смысловая, стилистическая правка, проверка терминологии.

Все эти действия основаны на глубоком понимании механизмов смыслообразования, текстопорождения и текстовосприятия, коммуникативно-прагматических факторов, влияющих на функционирование текста, логико-семантических связей, образующих смысловой план текста, и многих других лингвистических и экстралингвистических характеристик речевого сообщения, которые обычно и являются предметом переводческого анализа текста. Представители отрасли и научное сообщество часто подчеркивают важность одних и тех же текстовых параметров, но называют их по-разному, соответственно, создается поверхностное впечатление о том, что переводческий анализ текста в производственной деятельности не используется:

\begin{tabular}{|l|l|}
\hline $\begin{array}{c}\text { Терминология сайта } \\
\text { переводческой компании } \\
\text { Логрус Глобал }\end{array}$ & \multicolumn{1}{|c|}{$\begin{array}{c}\text { Терминология } \\
\text { теории перевода }\end{array}$} \\
\hline $\begin{array}{l}\text { «для кого предназначен } \\
\text { текст» }\end{array}$ & адресат перевода \\
\hline $\begin{array}{l}\text { «для чего предназначен } \\
\text { текст» }\end{array}$ & $\begin{array}{l}\text { коммуникативная цель ис- } \\
\text { ходного текста; коммуника- } \\
\text { тивная цель текста перевода }\end{array}$ \\
\hline «категория материалов» & $\begin{array}{l}\text { тип текста, функциональный } \\
\text { стиль }\end{array}$ \\
\hline
\end{tabular}

Среди прочего решение вопроса о том, насколько целесообразно применение машинного перевода и/ или постредактирования в отношении текста из кон- 
кретного заказа определяется переводческими компаниями на основе следующих факторов: 1) текст предназначен для привлечения клиентов / увеличения продаж; 2) текст предназначен для технических специалистов клиента (обеспечить использование оборудования персоналом); 3) текст предназначен для ознакомления заказчиком; 4) текст предназначен для внутреннего пользования. Таким образом, перед отправкой заказа в работу по существу производится переводческий анализ текста, даже если он так не называется. Этот анализ может быть беглым, спонтанным, не основанным на каких-либо строго определенных критериях, но, тем не менее, он занимает важное место в работе переводческой компании, потому что напрямую связан с качеством предоставляемой услуги.

Отдельного внимания заслуживает вопрос о том, кто именно занимается анализом текста в переводческой компании. В академических кругах традиционно считается, что аналитической компетенцией должен владеть специалист, непосредственно выполняющий письменный перевод. Именно поэтому в процессе обучения переводческий анализ включен в курсы письменного перевода, а стратегия обучения представляет собой двухступенчатый процесс, в котором сначала происходит ознакомление с содержанием текста и его предпереводческий анализ, а затем выполняется письменный перевод предложенного фрагмента или целого текста. Такая последовательность обусловлена дидактически и имеет большое значение для подготовки будущего специалиста, так как способствует развитию умения принимать осознанные переводческие решения и обеспечивать качество перевода. Однако в «полевых» условиях последовательность действий и состав исполнителей выглядят по-другому, что, вероятно, и вызывает сомнение в целесообразности переводческого анализа среди представителей отрасли. Способы организации работы существенно изменились с применением машинного перевода. Так, работая в команде под управлением менеджера проекта, переводчики имеют дело не с целым текстом, а с его фрагментами. «Переводчики теперь не имеют доступа к полному тексту и не имеют представления о проекте в целом. У них нет возможности принимать решения, предусматриваемые Skopos-теорией, так как о целях коммуникации им мало что известно. По сути, всеми вопросами стратегического планирования теперь занимается менеджер проекта или, возможно, маркетолог, поскольку проект в целом как совокупность текстов контролируется технологией в рамках общей системы управления» [9, с. 183]. Таким образом, функция анализа текста передается менеджеру проекта, редактору, в отдельных случаях
- маркетологу, которые должны уметь анализировать текст с целью принятия конкретных производственных и переводческих решений, т. е. знать особенности тех или иных специализированных текстов, запросы аудитории, условия рынка и принимающей культуры, и делать это достаточно быстро, не выделяя на данную процедуру дополнительные временные ресурсы.

Еще одним важным аргументом в защиту аналитической компетенции в современных условиях рынка лингвистических услуг является развитие языковой личности переводчика, проявление которой не исключается и при использовании машинного перевода. Действительно, ее проявление можно проследить во всех формах взаимодействия человека и технических средств, включая автоматический перевод. При автоматическом переводе с предредактированием происходит адаптация языковой компетенции переводчика к ограничениям применяемой системы перевода; в процессе автоматического перевода с интерредактированием переводчик решает отдельные лексико-грамматические задачи в ходе самого перевода; при автоматическом переводе с постредактированием языковая личность переводчика проявляется в полной мере, так как результирующий текст переводчик вправе редактировать любыми способами. Однако «при любой стратегии машинного перевода когнитивный и мотивационный уровни языковой личности переводчика реализуются в полной мере» $[10$, c. 192]. Таким образом, понятие языковой личности переводчика (в широком смысле - специалиста переводческой компании, включая менеджера проекта, редактора и др.) видоизменяется и уточняется с учетом современных реалий отрасли, но остается по-прежнему актуальным.

Актуальность компетенции возрастает с уровнем качества, предъявляемым к постредактированию машинного перевода. Аналитический центр Taus (Translation Automation User Society) выделяет 2 уровня качества постредактирования: приемлемое (следует точно передать смысл исходного сообщения, при этом не обязательна грамматическая, синтаксическая и стилистическая правильность); качество, отвечающее предъявляемым к публикации требованиям (точная передача смысла, понятность, соответствие нормам стиля, грамматики и синтаксиса) [11]. Действия редактора по обеспечению правильной передачи смысла (оба уровня качества) и соответствия нормам стиля (уровень качества, отвечающего предъявляемым к публикации требованиям) предполагают аналитическую компетенцию, что еще раз подтверждает необходимость сформированной способности текстового анализа у специалиста по оказанию лингвистических услуг. 


\section{Результаты исследования}

На основании вышеизложенного приходим к следующим выводам. Разнонаправленные тенденции, которые можно наблюдать в оценке аналитической компетенции переводчика у представителей вузов и отрасли, не говорят о ее неактуальности. Работодатели в полной мере пользуются аналитической компетенцией, но не осознают и не формулируют ее важность, в то же время вузы зачастую развивают эту компетенцию в отрыве от современных особенностей отрасли. Для того чтобы преодолеть это противоречие, необходима коррекция индикаторов компетенции (знать - уметь - владеть) на этапе обучения переводчика с учетом требований и ожиданий отрасли, включая умения, необходимые при использовании машинного перевода. При этом стоит учитывать, что переводческий анализ нужен в профессиональной деятельности не только переводчикам, но и менеджерам проектов, специалистам, ответственным за качество перевода, редакторам.

\section{ЛИТЕРАТУРА}

1. Бондаренко А. Ожидания научного сообщества и реалии отрасли // Профессиональный перевод и управление информацией. 2016. № 1. С. 37-45.

2. Никитина T. Г. Задачи формирования навыков переводческого анализа текста и редактирования в профессиональной подготовке переводчиков // Концепт. 2018. № V2. URL: http://e-koncept.ru/2018/186019.htm

3. Каширина Н. А. Предпереводческий анализ текста как фактор повышения качества письменных переводов // Известия ЮФУ. Технические науки. 2004. № 1 (36). С. 258.

4. Кашкин В. Б. Анализ текста в подготовке переводчика // Материалы XL Междунар. филол. конф., 14-19 марта 2011 г. : актуальные проблемы переводоведения / под ред. В. И. Шадрина. СПб. : Филол. ф-т СПбГУ, 2011. С. 49-57.

5. Pym A. Translation skill-sets in a machine-translation age // Meta : Journal des traducteurs / Meta : Translators' Journal. 2013. № 58 (3). Pp. 487-503. URL: https:// doi:10.7202/1025047ar

6. Княжева E. A. Переводческий анализ текста и качество перевода // Вестник Воронеж. гос. ун-та. Сер.: Лингвистика и межкультурная коммуникация. 2012. № 1. С. $170-174$.

7. Сдобников В. В. Стратегия перевода : общее определение // Вестник Иркут. гос. лингв. ун-та. 2011. № 1. C. $165-172$.

8. Шереметьева С. О., Калинникова О. И. Экспериментальный анализ индикаторов переводимости экономических текстов // Вестник ЮУрГУ. Сер.: Лингвистика. 2019. Т. 16, № 2. С. 44-50.

9. Пим Э. Теоретические парадигмы в переводоведении. СПб. : Изд-во С.-Петерб. ун-та, 2018. 255 с.

10. Бабина О. И. Языковая личность переводчика и машинный перевод // Вестник Челяб. гос. ун-та. Фило- логия. Искусствоведение. 2011. № 24 (239), вып. 57. C. 191-193.

11. TAUS. Machine Translation Post-editing Guidelines. URL: https://www.taus.net/think-tank/best-practices/ postedit-best-practices/machine-translation-post-editingguidelines

\section{REFERENCES}

1. Bondarenko A. Ozhidaniya nauchnogo soobshhestva i realii otrasli [Scientific community expectations and the real state of the field. In: Professionalnyj perevod i upravlenie informatsiej. 2016. No. 1. Pp. 37-45.

2. Nikitina T. G. Zadachi formirovaniya navykov perevodcheskogo analiza teksta i redaktirovaniya $\mathrm{v}$ professionalnoj podgotovke perevodchikov [Skills development for the translation text analysis and editing in translators professional training. In: Kontsept. 2018. No. V2. Available at: http://e-koncept.ru/2018/186019.htm

3. Kashirina N. A. Predperevodcheskij analiz teksta kak faktor povysheniya kachestva pismennykh perevodov [Pre-translation text analysis as a factor of the translation quality improvement]. In: Izvestiya YUFU. Tekhnicheskie nauki. 2004. No. 1(36). P. 258.

4. Kashkin V. B. Analiz teksta v podgotovke perevodchika [The text analysis in a translator's training] / V. B. Kashkin. In: Materialy XL Mezhdunar. filol. konf., 14-19 March 2011: aktual. probl. perevodovedeniya / ed. by V. I. Shadrina. SPb.: Filol. d-t SPbGU, 2011. Pp. 49-57.

5. Pym A. Translation skill-sets in a machine-translation age. In: Meta: Journal des traducteurs / Meta: Translators' Journal. 2013. No. 58 (3). Pp. 487-503. Available at: https:// doi:10.7202/1025047ar

6. Knyazheva E. A. Perevodcheskij analiz teksta i kachestvo perevoda [Translation text analysis and the translation quality]. In: Vestnik VGU. Seriya: Lingvistika i mezhkulturnaya kommunikatsiya. 2012. No. 1. Pp. 170-174.

7. Sdobnikov V. V. Strategiya perevoda: obshhee opredelenie [Translation strategy: the general definition]. In: Vestnik Irkutskogo gosudarstvennogo lingvisticheskogo universiteta. 2011. No. 1. Pp. 165-172.

8. Sheremetyeva S. O., Kalinnikova O. I. Ehksperimentalnyj analiz indikatorov perevodimosti ehkonomicheskikh tekstov [The experimental analysis of translatability indicators of economic texts]. In: Vestnik YUUrGU. Seriya: Lingvistika. 2019. Vol. 16, No. 2. Pp. 44-50.

9. Pim Eh. Teoreticheskie paradigmy v perevodovedenii [Theoretical paradigms in translation studies]. SPb.: SPbGU pub. house, 2018. $255 \mathrm{p}$.

10. Babina O. I. Yazykovaya lichnost perevodchika i mashinnyj perevod [The translator's linguistic personality and machine translation]. In: Vestnik Chelyabinskogo gosudarstvennogo universiteta. Filologiya. Iskusstvovedenie. 2011. No. 24 (239). Issue 57. Pp. 191-193.

11. TAUS. Machine Translation Post-editing Guidelines. Available at: https://www.taus.net/think-tank/ best-practices/postedit-best-practices/machine-translation-post-editing-guidelines 
Российский государственный педагогический университет имени А. И. Гериена

Елизарова Л. В., кандидат филологических наук, дочент кафедры перевода

E-mail: lyudmilaelizarova@yandex.ru

Кустова О. Ю., кандидат филологических наук, дочент кафедры перевода

E-mail: o_kustova@mail.ru

Поступила в редакциню 2 сентября 2020 г.

Принята к публикаџии 29 декабря 2020 г.

\section{Для цитирования:}

Елизарова Л. В., Кустова О. Ю. Анализ текста в условиях автоматизации переводческой деятельности // Вестник Воронежского государственного университета. Серия: Лингвистика и межкультурная коммуникация. 2021. № 1. C. 57-64. DOI: https://doi.org/10.17308/ lic. $2021.1 / 3238$
Herzen State Pedagogical University of Russia

Elizarova L. V., Candidate of Philology, Associate Professor of the Translation and Interpreting Department E-mail: lyudmilaelizarova@yandex.ru

Kustova O. Yu., Candidate of Philology, Associate Professor of the Translation and Interpreting Department E-mail: o_kustova@mail.ru

Received: 2 September 2020

Accepted: 29 December 2020

\section{For citation:}

Elizarova L. V., Kustova O. Yu. The text analysis in translation process automation. Proceedings of Voronezh State University. Series: Linguistics and Intercultural Communication. 2021. No. 1. Pp. 57-64. DOI: https://doi. org/10.17308/lic.2021.1/3238 